\title{
BEC Blossoms at EPS-10
}

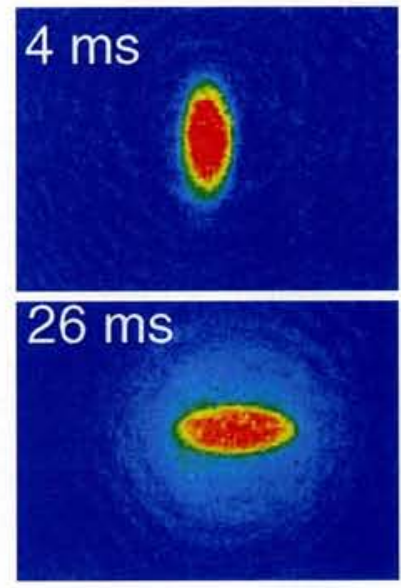

J.T.M. Walraven, who chaired the EPS-10 symposium Bose-Einstein Condensation, reports that one year after its first observation at JILA in Boulder, Colorado, Bose-Einstein condensation (BEC) in dilute gases continues to amaze the physics community. The three groups (all US based) which are presently collecting BEC data came to EPS-10 to establish the state-of-theart. If one thing became clear, then it is that BEC is shaking up the field of boson quantum fluids, after liquid helium- 4 has dominated the scene for more than half-a-century.

Bose-Einstein condensation (BEC) is achieved by evaporative cooling of magnetically trapped gas clouds, pre-cooled optically to sub-mK temperatures. The groups that invested some three years ago in evaporative cooling of alkali gases are today presenting their results. By now, BEC has been observed in ${ }^{87} \mathrm{Rb},{ }^{23} \mathrm{Na}$ and ${ }^{7} \mathrm{Li}$ at transition temperatures $\left(T_{c}\right)$ ranging from 200 $\mathrm{nK}$ to $2 \mu \mathrm{K}$, with up to about $10^{7}$ atoms at the onset of BEC. Basic thermodynamic properties, such as condensate fraction and heat capacity are as expected.

What is new? Not surprisingly, much effort has been put in improving apparatus. It has paid-off. This spring, W. Ketterle's group at MIT showed [ EN 27 (1996) 105] that dark-field imaging with far detuned light, a well-known technique from optical microscopy, enables (in situ) non-destructive detection of trapped gases - an essential step forward. Up to that point BEC could only be observed destructively by switching-off the trap, while observing the expanding gas cloud using optical absorption. Further, the accuracy of density and temperature determinations was greatly improved. On top of this quantitative progress, the true news came as refreshingly new qualitative results.

\section{New Qualitative Results}

Carl Wieman (JILA) presented the first results obtained for ${ }^{87} \mathrm{Rb}$ with the new JILA apparatus ("double MOT-baseball trap"). Most exciting in his talk was the demonstration of two coexisting condensates, differing in internal state, hyperfine state $\left(\mathrm{F},{ }_{\mathrm{m}} \mathrm{F}\right)=(2,2)$ versus $(1,-1)$. The two condensates were seen to displace each other rather than to merge. This is quite remarkable for gas clouds with atomic mean-free paths larger than their size! Stronger evidence for interacting condensed matter behaviour in these dilute gases (density $<10^{15}$ atoms $/ \mathrm{cm}^{3}$ ) is hard to imagine. Another novelty was that the $(1,-1)$ component of the

J.T.M. Walraven, Professor of Physics at Amsterdam University, was appointed recently as the Director of the FOM-Institute for Atomic and Molecular Physics (AMOLF), Amsterdam.

Two-dimensional probe absorption images of a Bose-Einstein condensate of $5 \times 10^{623} \mathrm{Na}$ atoms showing oscillation between two elongated shapes (about $150 \mu \mathrm{m}$ in length) for the inner condensed phase. The outer (normal) fraction expands isotropically like a classical gas. The times-offlight after the trap is switched off are indicated. Courtesy of W. Ketterle.

gas was sympathetically cooled into the $\mathrm{BEC}$ regime through collisions with the evaporatively cooled $(2,2)$ component, a process in which only $50 \%$ of the $(1,-1)$ atoms was lost while the $(2,2)$ population was decimated by a factor 60 . Further, the JILA group found that collective excitations persist longer in Bose condensed than in non-Bose condensed phases. Clearly, the JILA group is entering deeper waters.

Comparing the quantum gases, an important figure-of-merit is the s-wave scattering amplitude $a$ which embodies the overall effect of the elastic scattering potential. In ${ }^{87} \mathrm{Rb}$ and ${ }^{23} \mathrm{Na}$ the scattering amplitude is positive. This corresponds to an effectively repulsive interaction. In contrast, ${ }^{7} \mathrm{Li}$ has a negative $a$. The consequences of the sign of $a$ have been debated widely. It is well-known that for kinetic effects, such as evaporative cooling, the sign of $a$ is irrelevant since the collisional cross-section depends on $a^{2}$. For energy considerations above $T_{c}$, the sign of the scattering amplitude is also of little consequence as thermal agitation easily dominates over the effect of interactions in these dilute gases. However, a condensate exhibits zero-temperature behaviour and interaction effects will dominate as soon as they exceed the minute zero-point vibrational energy (typically 5 nanokelvin) in the trap. This effect determines the actual size of a condensate in a confining potential.

Boudewijn Verhaar (Eindhoven University of Technology) reported on refined methods to obtain accurate information on the interaction potentials of alkali systems. $A b$ initio calculation of the scattering amplitude is not possible except for the lightest elements. For the heavy alkalis, even the sign of $a$ is hard to establish without input of experimental information. Photo-association spectra can provide such input. For example, the scattering amplitude of ${ }^{7} \mathrm{Li}$ can be determined to within $2.5 \%$ using two-photon photo-association data. In his talk at EPS-10, Verhaar discussed how the interaction potential in ${ }^{87} \mathrm{Rb}$ can be pinned down through the investigation of shape resonances (associated with virtual bound states) in the dwave scattering channel. He also discussed how with the aid of a magnetic field the relative importance of the singlet and triplet contributions to the scattering amplitude in hyperfine mixed spin channels can be changed to tune the value of $a$. The Eindhoven group found that for realistic fields, in the case of ${ }^{39} \mathrm{~K}$ and ${ }^{41} \mathrm{~K}$, this can give rise to Feshbach resonances in which the scattering cross-section diverges.

Randy Hulet (Rice University) reported on his work with $\mathrm{Li}$. This has been somewhat controversial owing to the static trap (permanent magnets) used by this group, since diagnostics have proven to be tricky. Both at JILA and MIT the convincing initial experiments were done by investigating expanding gas clouds using light absorption after switching off the trapping field. This is not possible at Rice. This summer, Hulet, following Ketterle's nondestructive approach, carefully analyzed microscope imaging techniques and found phase-contrast imaging to be the most well-suited for obtaining reliable density and temperature data. With the experiments at Rice, the basic phenomenology of $\mathrm{BEC}$ in quantum gases with negative $a$ now seems to be well established and in line with existing theory: as long as the occupation of the oscillator states in the trap remains small, the kinetic energy due to confinement will overcome the attractive interaction and a metastable condensate can form. Beyond a certain occupation, for the conditions at Rice typically 1500 atoms, the attractive interactions start to dominate and the condensate becomes unstable. It will be a challenge for future experiments to reveal the kinetics that limit the conden- 
sate occupation to this upper level, and to study the collective excitations in such an unusual quantum fluid.

Wolfgang Ketterle (MIT) presented the results obtained with his improved apparatus, in which some $5 \times 10^{6}{ }^{23} \mathrm{Na}$ atoms can be Bose condensed within a $30 \mathrm{~s}$ cooling time and in which the MIT group introduced the dark-field imaging method. An ingenious "clover leaf" coil geometry permits full optical access while offering the advantages of a Ioffe-Pritchard trap. The samples have a long, elongated shape so that the mean-free path in the axial direction can be made smaller than the sample size. This gives access to the hydrodynamic regime in which the free expansion of the gas is limited by the speed of sound. On collectively exciting the condensate, the normal mode frequencies were found to be in accordance with mean-field theory. As in the JILA experiments, the big issue here is to understand the damping.

Some of the most exciting aspects of the field at the present stage are of course the "simple" exploratory experiments. Ketterle mentioned several of them: cutting the condensate in two parts with a thin sheet of far detuned light and looking for interference fringes when the parts meet -

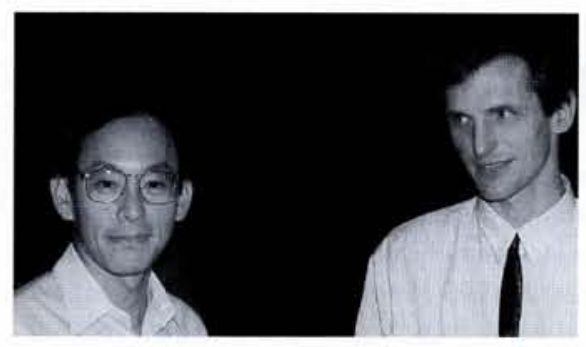

W. Ketterle (MIT), on the right, with S. Chu (Stanford University) at EPS-10.

the cutting works but the fringes are not (yet) observed. Then, addressing the quest for the atom laser, Ketterle showed how an RF pulse can be used as an output coupler to extract beams of $(1,1)$ and $(1,0)$ atoms from the condensate. Also here the search for coherence has still to begin.

\section{Some Definite Trends}

What are the trends? Currently, all experiments are done with Ioffe quadrupole magnetic field geometries. This type of trap offers large flexibility in the choice of trap parameters such as minimum field, axial and radial frequencies, etc. Also, a good start has been made towards the ideal of accurate non-destructive detection, although there is room for improvement in spatial resolution, and for three-dimensional imaging. Looking at the future it is interesting to note that, thus far, the BEC phase line has only been crossed during cool-down by evaporation. Clearly, a next step will be to develop methods to cross the phase line reversibly. This would be a good starting point to investigate, for instance, the kinetics of Bose condensate formation, something simply inconceivable with highdensity condensed matter. Another deep issue to understand will be the damping of collective excitations of the condensate which cannot be understood on the level of mean-field theory.

From the condensed matter side, there is a desire to watch the nucleation of a vortex in a condensate. From the quantum optics side there is a strong push to couple a coherent beam of atoms out of the condensate, aiming for the atomic equivalent of the laser. Or better still, look at phase relations between two independently created condensates. On the theory side, as Franck Laloë discusses below, the quantum opticians confront settled opinions of condensed matter theorists. It will be interesting to watch what both communities will contribute to the understanding of these dilute little gas clouds of common interest.

\section{Is Particle Number Conservation Broken Spontaneously in Bose Condensates?}

\begin{abstract}
Franck Laloë from the Ecole Normale Superieur reports that a particularly lively discussion on the question of spontaneous symmetry breaking in Bose-Einstein condensates took place during an improvised session at a recent Les Houches workshop on Collective effects in ultracold atomic gases (the workshop was organized by Christophe Salomon and Yvan Castin and attended by about 80 physicists from all over the world).
\end{abstract}

One refreshing characteristic of the field of ultracold atomic gases is that it brings together different scientific communities: condensed matter physicists who have been familiar with superfluid systems and their various theoretical aspects for years, and specialists in atomic physics and quantum electronics who have been busy with various properties of dilute gases for a long time, while superfluidity is of course something new. These fields have different traditions and cultures, and very naturally the newcomers to superfluids tend to question some concepts which are well established in condensed matter physics. One of them, which indeed gave rise to lively discussions between different points of view at the recent Les Houches workshop, is spontaneous symmetry breaking of particle number conservation.
On the one hand, some condensed matter physicists consider this idea as completely indispensable for understanding the essence of superfluidity, while on the other hand some atomic physicists say that they could count the number of atoms in their traps so that invoking a violation of particle number conservation is more akin to magic than good physical reasoning. As always, a continuum of intermediate opinions between the two extremes is possible.

\section{Symmetry Breaking}

Initially, the idea of spontaneous symmetry breaking was introduced in the 1960 s by analogy with ferromagnetism [see, for instance, P.W. Anderson, Basic notions of condensed matter physics (Benjamin, 1984); or P. C. Hohenberg \& P.C. Martin, Annals of Physics 34 ( 1965) 291].
In spin ferromagnetics, the spin Hamiltonian is perfectly isotropic (the Hamiltonian commutes with angular momentum) so that there seems to be no preferred direction in space for a macroscopic magnetization to appear. Still we know that, in practice, below the transition temperature, all ferromagnetic systems "choose a direction in space" in order to develop a macroscopic magnetization; rotation invariance is broken. For superfluids, the symmetry is no longer invariance under space rotation, but invariance under a change of the phase of the wave function. This is related to the commutation of the Hamiltonian with the total number of particles and corresponds to a different invariance group than rotation, but otherwise the basic idea is the same. The analogue to the magnetization is the mean value of the quantum field operator, often called a "macroscopic wave function"; the latter can have non-zero values only if the system is in a coherent superposition of states with different numbers of particles. Another similar physical phenomenon is the onset of oscillations in a laser going through threshold; although lasers are not physical systems at equilibrium, when the oscillation starts, the system also "chooses a phase" in a process which is also analogous to the appearance 